\title{
Nonlinear Robust Observer-Based Fault Detection for Networked Suspension Control System of Maglev Train
}

\author{
Yun Li, Guang He, and Jie Li \\ College of Mechatronics Engineering and Automation, National University of Defense Technology, Changsha 410073, China \\ Correspondence should be addressed to Guang He; heguang410@163.com
}

Received 11 January 2013; Revised 22 April 2013; Accepted 26 April 2013

Academic Editor: Engang Tian

Copyright (C) 2013 Yun Li et al. This is an open access article distributed under the Creative Commons Attribution License, which permits unrestricted use, distribution, and reproduction in any medium, provided the original work is properly cited.

\begin{abstract}
A fault detection approach based on nonlinear robust observer is designed for the networked suspension control system of Maglev train with random induced time delay. First, considering random bounded time-delay and external disturbance, the nonlinear model of the networked suspension control system is established. Then, a nonlinear robust observer is designed using the input of the suspension gap. And the estimate error is proved to be bounded with arbitrary precision by adopting an appropriate parameter. When sensor faults happen, the residual between the real states and the observer outputs indicates which kind of sensor failures occurs. Finally, simulation results using the actual parameters of CMS-04 maglev train indicate that the proposed method is effective for maglev train.
\end{abstract}

\section{Introduction}

Maglev train is a new type of track transportation vehicle with no need of traditional wheels, which levitates the vehicle on the tracks using electromagnetic force. With the advantages of low noise, no pollution, small turning radius, and ease of maintenance, Maglev train is considered to be suitable for urban transportation and has extensively been studied $[1,2]$. The suspension control system is the pivotal component of the electromagnetic suspension system, which is inherently instable and nonlinear, and it is necessary to design feedback controllers for adjusting the electromagnetic forces between the electromagnets and the tracks to achieve stable levitation. Considering of the exiting complicated electromagnetic interference (EMI) in the suspension control system, the networked control system is adopted to avoid the electromagnetic interference with wires and improve the reliability of data transmission [3, 4]. By doing this, it can also realize data sharing and communication among suspension modules on the train and is helpful for the control of the suspension modules.

The networked suspension control system mainly consists of three parts [5]: the sensor group, the controller, and the electromagnets acting as actuators. In engineering practice, high security and reliability, especially high reliability of the suspension control system, are required for the application of Maglev trains. If any fault happens, the suspension control system may turn to be unstable, which endangers the vehicle's operation. It is proved by researches and statistics that sensor faults eventually become the major cause for the invalidation of suspension control system [6]. Meanwhile, considering the existing network-induced time delay in the data transmission, it is more complex and more difficult to separate and recognize the faults of the sensors in networked control system than doing the job in other control system. Therefore, it is of great theoretical and practical value to develop the researches on the faults detection in networked suspension control system.

For the present, lots of the researches have been focused on the modeling, controllers design, and stability of the networked control system [7-10]. In recent years, fault detection and diagnosis on networked control systems has also been regarded as an important research direction for engineering requirements. Wang et al. [11] adopted reference model approaches to deal with the fault detection of the networked control systems with random and unknown networkinduced delay that might be larger than one sampling period. In [12], Liu et al. study fault detection of linear systems over 
networks with bounded packet loss, and a fault detection filter for switched system is designed to satisfy performance requires. Zhang et al. [13] proposes the approach of minimum error entropy filter to indicate the existing fault in the networked control systems with random delays, packet dropout, and noises by the residual generated by the filter. In [14], T$S$ fuzzy model is adopted to deal with the fault detection of the networked control system with Markov transfer delays. Besides, observer-based approaches have also been applied to fault detection of the networked control system. As for a class of nonlinear networked control systems (NCSs) with Markov transfer delays, a novel sliding mode observer approach [15] is proposed to solve the fault estimation problem. He et al. [16] study the problem of robust fault detection for a nonlinear networked control system model approximated by uncertain T-S fuzzy models. Wherein the residual obtained from the robust fault observer can be sensitive to the fault but robust to exogenous disturbance. In [17-19], for several networked control systems, respective observer-based fault detection approaches have been developed. Tian et al. [20] concerns the fault tolerant control for discrete networked control systems (NCSs) with probabilistic sensor and actuator fault. By using Lyapunov functional method and linear matrix inequality technology, sufficient conditions for the mean square stable (MSS) of the NCSs can be obtained. The literature [21] studies the nonlinear networked control system with T-S fuzzy model, and the Lyapunov functional and the linear matrix inequality (LMI) are applied to develop two new stability conditions. These conditions and an algorithm are used to design a controller to achieve robust mean square stability of the system. The literature [22] investigates robust and reliable $H_{\infty}$ filter design for a class of nonlinear networked control systems; four new theorems are proved to cover the conditions for the robust mean square stability of the systems under study in terms of LMIs, and a decoupling method for the filter design is developed.

The networked suspension control system is nonlinear, and a precise mathematical model is always difficult to be obtained in a practical system for the existing model error, time delay, and unknown disturbance. Therefore, designing the fault detection methods with the property of robust and insensitive to uncertain disturbance turns to be an important research direction for the networked suspension control system. At present, an observer-based approach is considered to be one of the most promising fault detection methods that have excellent application potential for nonlinear networked control system. In [23], an adaptive nonlinear observer, which can dominate the effects of unmodeled dynamics independently to prevent the state estimations from diverging and to get the precise estimations, is designed. However, it is validated that the networked suspension control system cannot satisfy the applied condition of this adaptive nonlinear observer. By promoting the application domain of the observer introduced by [23], we present a nonlinear robust fault observer to handle the fault detection of the networked suspension control system. A novel nonlinear robust observer with the compensator for the effects of network delay and uncertainty of the system is proposed by adaptive method, and the state estimate error is proved to be bounded and with arbitrary precision by adopting an appropriate Lyapunov function. When sensor faults happen, the residual between the real states and the observer outputs indicates the direction and magnitude of the sensor fault. Different from the existing method in dealing with the linear networked suspension control system, the proposed method concerns the strong nonlinear characteristic in the system modeling and random induced time delay.

The overall purpose of this paper is to develop a nonlinear robust fault observer for the fault detection of the networked suspension control system. The rest of the paper is organized as follows. Section 2 introduces the networked suspension control system of Maglev train with CAN bus serving as the information transmission network of the system and also analyzes the possible sensor failures. Section 3 designs a nonlinear robust fault observer, and the estimate error is proved to be bounded by adopting an appropriate Lyapunov function. And the residual between the real states and the observer outputs indicates the sensor failures. Simulations in Section 4 using the actual parameters of CMS-04 Maglev train demonstrate the effectiveness of this method. Finally, Section 5 gives the conclusion of this paper.

\section{Mathematical Model of the Networked Suspension Control System}

In a Maglev train, the suspension control system is the key part. Its normal working is the premise that the train can run in safety. The suspension system and motor propulsion system cause an adverse electromagnetic environment of control field of the Maglev train. The analogue transmission method applied to the signal of the control system is easy to cause "glitch" to signal conductors due to external disturbance, which may cause a serious impact on the system's suspension control function. Therefore, a communication network is to be introduced in the suspension control system to realize the digital network transmission of signals, which may ensure that the signals can transmit effectively so as to avoid the impact of electromagnetic interference.

The networked sensors system design is adopted as the general scheme in the networked suspension control system. Network nodes of the sensor collect the information of system status with a constant frequency and transmit it, after digitization of data, to corresponding control nodes through CAN bus network. As for the controllers working in an eventdriven model, once the data of sensors reach the node of a controller through the network, the node starts computation immediately to get the control variables and send it to the electromagnet in a shape of PWM wave to form a closed loop. The PMW wave itself is capable of resisting disturbance, and the control quantity needs no network transmission. And the structure of Maglev network control system is shown in Figure 1.

The single suspension node is the basic suspension unit of Maglev train. Therefore, the analysis and simulations in this paper are based on the single suspension node. Figure 2 shows the scheme of the networked suspension control system (single node). The whole system consists of the suspension 


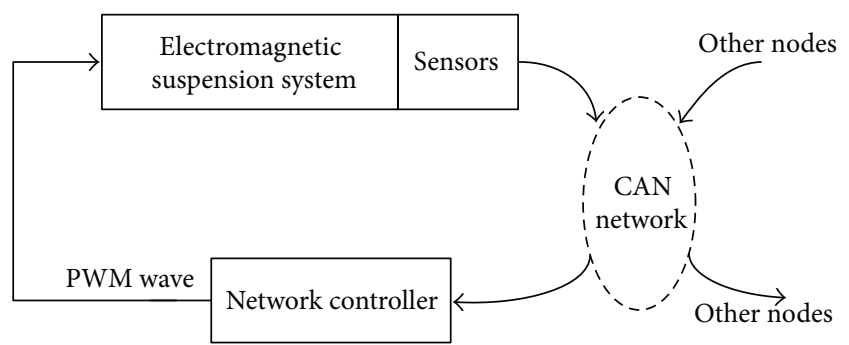

Figure 1: The structure of networked suspension control system.

network controller, integrative sensors, the network, the wave chopper, and the electromagnets. Especially, the network can link to other nodes and their corresponding upper surveillance and control layers.

A network structure is used for the Maglev control system to strengthen the system's capacity restraining strong disturbance and unavoidably brings some problems such as delay of signal transmission and loss of network data packages $[23,24]$. Such problems may degrade the performance of the suspension control system and even make the system unstable under adverse circumstance. Therefore, it is necessary to consider the impact of the network on the control system. First we make assumptions as follows for the system.

(1) The time-driven model is used for nodes of the network sensors and the event-driven model for the controllers.

(2) The network-induced time delay satisfies $0 \leq \tau \leq T$.

(3) There are three types of status information-gaps, current, and speed-transmitted from the sensors' nodes to the network.

According to the data stream path in the CAN buscontrolled network, the message delay time of the CAN bus is

$$
\tau=t_{\text {sent }}+t_{\text {wait }}+t_{\text {rev }},
$$

where $t_{\text {sent }}, t_{\text {wait }}$, and $t_{\text {rev }}$ are transform processing delay, CAN bus access waiting delay, and receiving processing delay, respectively. Then, $\tau$ will be a parameter with a supreme when the CAN network is not busy. This assumption is proved reasonable by engineering practice $[3,25]$.

Denote downward as positive. Let the electric resistance of electromagnet be $R$; inductance, $L_{i}$; voltage of two ends of electromagnet, $u$; current, $i$; mass of bogie and carriage, $m$; and acceleration of gravity, $g$. Assume that the unknown disturbance force applied on the system is $d(t)$ and define that the status variable of the system is $x=\left(x_{1}, x_{2}, x_{3}\right)^{\prime}$, where $x_{1}=z$ is the suspension gap of the system, $x_{2}$ is the system's vertical speed that can be obtained by integration of acceleration transducer, and $x_{3}=i$ is the current intensity flowing through the electromagnet. In accordance with the literature [26], the dynamic equation of the suspension control system can be expressed as follows:

$$
\dot{x}=f(x)+B u(x, t-\tau)+d(t),
$$

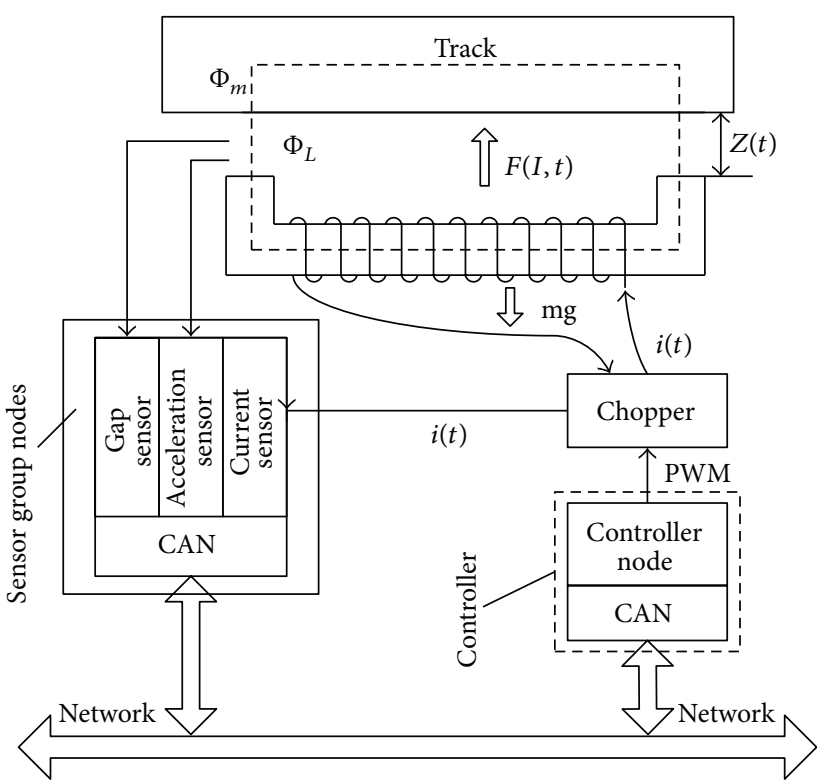

FIGURE 2: The scheme of the networked suspension control system (single node).

where $f(x)=\left[\begin{array}{lll}x_{2} & -k x_{3}^{2} / m x_{1}^{2} & -(R / L) x_{3}\end{array}\right]^{T}, B=\left[\begin{array}{lll}0 & 0 & 1 / L\end{array}\right]^{T}$ is the nominal system model, $k$ are parameters relevant to suspension system structure, and $d$ represents the impact of uncertainty of parameterkon the system.

It is difficult to use the state equation (2) to analyze and design the control system. So we change its form to some extent. According to the working requirement, the current $i_{\text {eq }}$ of certain intensity flowing through the electromagnet generates the suspension force to make the suspension system maintain stablity at the balance position $z_{\mathrm{eq}}$. Accordingly, the balance point of the system is $x=\left(z_{\mathrm{eq}}, 0, i_{\mathrm{eq}}\right)$. Using Taylor formula at the balance point, the dynamic equation can be expressed as follows:

$$
\dot{x}=A x+B u(x, t-\tau)+f_{\text {h.o.t }}(x)+d(t),
$$

where $A=\left.(\partial f / \partial x)\right|_{x=\left(z_{\mathrm{eq}}, 0, i_{\mathrm{eq}}\right)}=\left(\begin{array}{ccc}0 & 1 & 0 \\ 2 g / z_{\mathrm{eq}} & 0 & -2 g / i_{\mathrm{eq}} \\ 0 & 0 & -R / L\end{array}\right)$, and $f_{\text {h.o.t }}(x)$ are the higher order terms by the system's Taylor expansion.

$f(x)$ is bounded and hence the $f_{\text {h.o.t }}(x)$ is bounded. $u(x, t-\tau)$ is the system's control output which makes the system (3) keep stable under the circumstance of certain network delay and meet certain performance requirement. Because the higher order term $f_{\text {h.o.t }}(x)$ was not deleted in the linearization process, the dynamic equation (2) is equivalent to (3). Therefore, the dynamic equation (2) is equivalent to (3).

Discrete the system (3), and we can get

$$
\begin{aligned}
x(k+1)= & A x(k)+B_{0}\left(\tau_{k}\right) u(k) \\
& +B_{1}\left(\tau_{k}\right) u(k-1)+B_{d} f_{\text {h.o.t }}(x)+B_{d} d(k),
\end{aligned}
$$


where

$$
\begin{aligned}
& x(k)=x(k T), \quad A=e^{A T}, \quad B_{d}=\int_{0}^{T} e^{A t} d t B, \\
& B_{0}\left(\tau_{k}\right)=\int_{0}^{T-\tau_{k}} e^{A t} d t B, \quad B_{1}\left(\tau_{k}\right)=\int_{T-\tau_{k}}^{T} e^{A t} d t B .
\end{aligned}
$$

Denote $B_{0}\left(\tau_{k}\right)$ and $B_{1}\left(\tau_{k}\right)$ as $B_{0}, B_{1}$, and we have the expression

$$
B_{0}=B_{0}+H F\left(\tau_{k}\right) E, \quad B_{1}=B_{1}+H F\left(\tau_{k}\right) E,
$$

where $B_{0}, B_{1}, H$, and $E$ are constant matrices. $F\left(\tau_{k}\right)$ is the function of $\tau_{k}$, which has $F^{T}\left(\tau_{k}\right) F\left(\tau_{k}\right) \leq I$. The proof and expression of $B_{0}, B_{1}, H$, and $E$ can be found in the literature [27]. Hence, the discrete model of networked Maglev control system can be expressed as

$$
\begin{aligned}
x(k+1)= & A x(k)+\left(B_{0}+\Delta B\right) u(k) \\
& +\left(B_{1}-\Delta B\right) u(k-1)+B_{d} f_{\text {h.o.t }}(x)+B_{d} d(k),
\end{aligned}
$$

where $\Delta B=H F\left(\tau_{k}\right) E$. Let $d_{f}(k)=\Delta B u(k)-\Delta B u(k-1)+$ $B_{d} f_{\text {h.o.t }}(x)+B_{d} d(k)$, and the system (7) can be rewritten as

$$
x(k+1)=A x(k)+B_{0} u(k)+B_{1} u(k-1)+d_{f}(k) .
$$

$x_{1}(k)$ is the output of the networked Maglev control system, so

$$
y(k)=x_{1}(k)=C x(k),
$$

where $C=[0,0,1]$. Since the system's status in the actual suspension system is bounded, we can always find the positive constants $\zeta$ and $\phi$ to make $d_{f}(k)$ satisfy the following equation:

$$
\left\|d_{f}(x, t)\right\| \leq \zeta\|x\|+\phi
$$

That is, $\zeta\|x\|+\phi$ defines the range of uncertainty and outside disturbance of the system model.

\section{Fault Detection Approach Based on a Nonlinear Robust Observer}

The sensors group is made up of gap sensors, current sensors, and acceleration sensors. In practice, it needs several gap sensors to make the Maglev train get across the joints existing in the tracks. By doing this, the gap sensors realize the redundancy in hardware. Therefore, the faults diagnosis of the sensors in suspension control system is focused on the current sensors and the acceleration sensors. The aim of fault detection is to recognize the direction and the extent of the sensor faults and produce an alarming signal. This method plays an important significance in improving the maintainability and reliability of the control system. If highly reliable gap signals can be used to estimate the correct current and velocity signals, a comparable big residual between the estimating current and speed signals output and the physical sensors will indicate that faults occur in the sensors. System (3) is a typical nonlinear system; therefore, designing the fault diagnosis methods with the property of robust and insensitive to uncertain disturbance turns to be an important research direction. In this section, a fault detection approach based on a nonlinear robust observer is proposed and designed.

Lemma 1 (see [28]). For given Hurwitz matrix $A_{0} \in R^{n \times n}$ and constants $v$ and $\varepsilon>0$, there exist positive definite and symmetrical matrix $P$ to ensure the validity of the following Riccati equation:

$$
A_{0}^{T} P+P A_{0}+v^{2} P P+|v| I<0 .
$$

Assumption 2. For $P$ in Lemma 1, there exist $E, h$ such that

$$
P E=C^{T} h,
$$

where $E$ and $h$ are constant matrices with appropriate dimensions.

Assumption 3. There exists a constant $\varphi$, which ensures the validity of the following Riccati equation:

$$
\|\varphi C e(k)\| \geq\|e\| .
$$

Theorem 4. Based on Assumption 2, one defines e $(k)=\widehat{x}(k)-$ $x(k)$, and then the estimation of the system's states from (15) of nonlinear observer is uniformly bounded. If one chooses appropriate gains of $\sigma>0$, the observation error of the observer can be made arbitrarily small:

$$
\begin{aligned}
\widehat{x}(k+1)= & A \widehat{x}(k)+B_{0} u(k)+B_{1} u(k-1) \\
& +E \beta+L(y(k)-C \widehat{x}(k)),
\end{aligned}
$$

where $L$ is the feedback coefficient to make $(A-L C)$ be a Hurwitz matrix, and $\beta$ is a scalar self-adaptive parameter that is expressed by the following equation:

$$
\begin{aligned}
\beta(k+1)= & -\sigma \beta(k)-h^{T} C e(k)-\alpha \operatorname{sgn}(\beta(k)) \\
& \times\left(\|\varphi C e(k)\|^{2}+\|\widehat{x}(k)\|^{2}\right) .
\end{aligned}
$$

Proof. From (8) and (14), we can obtain

$$
e(k)=\hat{x}(k)-x(k)=(A-L C) e(k)-d_{f}(x, k)+E \beta(k) .
$$

Select the lyapunov function:

$$
V(k)=\frac{1}{2}\left[e^{T}(k) P e(k)+\beta^{2}(k)\right] .
$$


The derivation of $V$ along the error equation (16) is written as

$$
\begin{aligned}
\Delta V(k)= & V(k+1)-V(k) \\
= & \frac{1}{2} e^{T}(k)\left[(A-L C)^{T} P+P(A-L C)\right] e(k) \\
& -e^{T}(k) P d_{f}(x, k) \\
& +e^{T}(k) P E \beta+\beta(k) \beta(k+1) \\
\leq & \frac{1}{2} e^{T}\left[(A-L C)^{T} P+P(A-L C)\right] e+\|P e\| \\
& \times(\xi\|x\|+\phi)+e^{T}(k) P E \beta(k)+\beta(k) \beta(k+1) .
\end{aligned}
$$

Based on $\|x\| \leq\|e\|+\|\widehat{x}\|$, we can obtain the following:

$$
\begin{aligned}
\Delta V(k) \leq & \frac{1}{2} e^{T}(k)\left[(A-L C)^{T} P+P(A-L C)\right] e(k) \\
& +\left\|e^{T}(k) P\right\|(\xi\|\widehat{x}(k)\|+\xi\|e(k)\|+\phi) \\
& +e^{T}(k) P E \beta+\beta(k) \beta(k+1) \\
\leq & \frac{1}{2} e^{T}(k)\left[(A-L C)^{T} P+P(A-L C)\right] e(k) \\
& +\xi\left\|e^{T}(k) P\right\| \cdot\|e(k)\|+\lambda_{\max }(P) \xi\|e\|\|\widehat{x}(k)\| \\
& +\lambda_{\max }(P) \phi\|e\|+e^{T}(k) P E \beta+\beta(k) \beta(k+1) \\
\leq & \frac{1}{2} e^{T}(k)\left[(A-L C)^{T} P+P(A-L C)\right. \\
& \left.+\frac{1}{2} I+\frac{1}{2} \xi^{2} P P\right] e(k) \\
& +\lambda_{\max }(P) \xi\|e(k)\|\|\widehat{x}(k)\|+\lambda \max (P) \phi\|e(k)\| \\
& +e^{T}(k) P E \beta(k)+\beta(k) \beta(k+1) .
\end{aligned}
$$

From Lemma 1, we can obtain

$$
\begin{aligned}
\Delta V(k) \leq & \lambda_{\max }(P) \xi\|e(k)\|\|\widehat{x}(k)\|+\lambda_{\max }(P) \phi\|e(k)\| \\
& +e^{T}(k) P E \beta(k)+\beta(k) \beta(k+1) .
\end{aligned}
$$

For any positive constant $\alpha, \lambda_{\max }(P) \phi\|e(k)\| \leq$ $(1 / 2 \alpha)\left(\lambda_{\max }^{2}(P) \phi^{2}+\alpha\|e(k)\|^{2}\right)$, and then we have

$$
\begin{aligned}
\Delta V(k) \leq & \frac{1}{2 \alpha|\beta(k)|} \lambda_{\max }^{2}(P) \xi^{2}+\alpha|\beta(k)|\|e(k)\|^{2}\|\widehat{x}(k)\|^{2} \\
& +\frac{1}{2 \alpha|\beta(k)|} \lambda_{\max }^{2}(P) \phi^{2}+\alpha|\beta(k)|\|e(k)\|^{2} \\
& +e^{T}(k) P E \beta(k)+\beta(k) \beta(k+1) .
\end{aligned}
$$

Substituting (15) into (21), we obtain

$$
\begin{aligned}
\Delta V(k) \leq & \frac{1}{2 \alpha|\beta(k)|} \lambda_{\max }^{2}(P) \xi^{2}+\alpha|\beta(k)|\|e(k)\|^{2}\|\widehat{x}(k)\|^{2} \\
& +\frac{1}{2 \alpha|\beta(k)|} \lambda_{\max }^{2}(P) \phi^{2}+\alpha|\beta(k)|\|e(k)\|^{2} \\
& +e^{T}(k) P E \beta(k)-\sigma \beta^{2}(k)-\beta(k) h^{T} \operatorname{Ce}(k) \\
& -\alpha|\beta(k)|\left(\|\varphi C e(k)\|^{2}+\|\varphi \operatorname{Ce}(k)\|^{2} \cdot\|\widehat{x}(k)\|^{2}\right) .
\end{aligned}
$$

From Assumption 2, we have

$$
\begin{aligned}
\frac{d V}{d t} & \leq-\sigma \beta^{2}+\frac{1}{2 \alpha|\beta|} \lambda_{\max }^{2}(P) \xi^{2}+\frac{1}{2 \alpha|\beta|} \lambda_{\max }^{2}(P) \phi^{2} \\
& =-\sigma \beta^{2}+\Omega,
\end{aligned}
$$

where $\Omega=(1 / 2 \alpha|\beta|) \lambda_{\text {max }}^{2}(P) \xi^{2}+(1 / 2 \alpha|\beta|) \lambda_{\max }^{2}(P) \phi^{2}$. Therefore, $V(e, \beta)$ is decreasing monotonously to a closed ball of $B=\left\{(e, \beta) \in R^{n} \times R: V(e, \beta) \leq \sigma^{-1} \Omega\right\}$, so $e$ and $\beta$ are generally and uniformly bounded. If we choose that $\sigma$ is small enough, the closed ball $B$ will be small enough. Thereby the error of the observer could be arbitrarily small.

If we let $C=[1,0,0]$ in (9), namely, the observed results of nonlinear observer only depending on the gap sensors, it can be seen that the observer can estimate correct system status information. Therefore, if faults occur in the current sensors or the acceleration sensors, the measured values of the states of the observers and physical sensors would be notably different. Define the threshold $\delta$. If

$$
|e| \geq \delta
$$

it indicates that the fault has occurred and according to the direction of $e$, we can further diagnose which one has the fault. It should be noted that due to the network delay, the estimated values of general status and measured values of the physical sensors are not exactly the same, so $\delta$ is slightly greater than 0 .

\section{Simulations and Results}

With CMS-04 Maglev train developed by the National University of Defense Technology as the object, the simulations have verified the effectiveness of the fault diagnose scheme. Table 1 shows the actual parameters of the Maglev system.

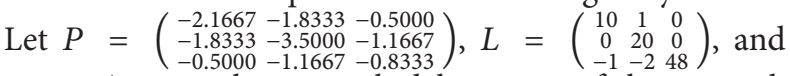
$\sigma=120$. Assume the network delay range of the network suspension system be $0 \leq \tau_{k} \leq T$, where $T=1 \mathrm{~ms}$. If there are no faults occurring in the sensors, the observer output diagram is shown in Figure 3. And the curves show levitation gap, the residual between the velocity obtained from observer and computing from the accelerometer output, and the residual between the current obtained from observer and current sensors, respectively. From Figure 3, the system is 


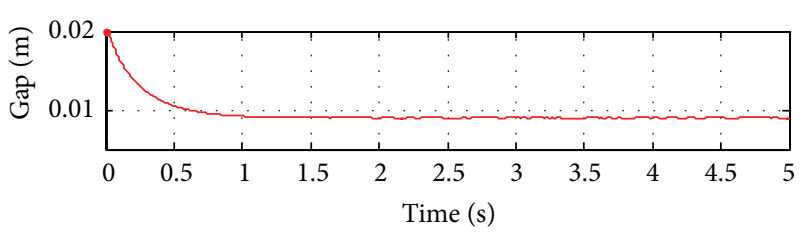

(a)

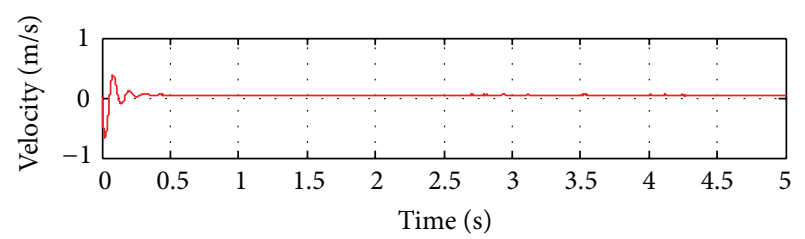

(b)

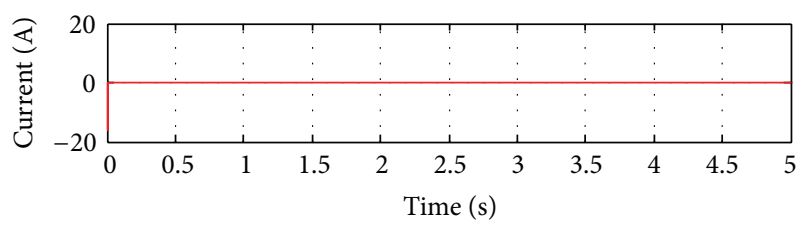

(c)

FIGURE 3: Output of robust fault observer with disturbance and time delay.

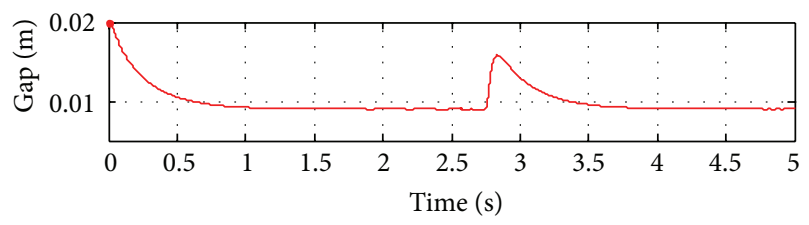

(a)

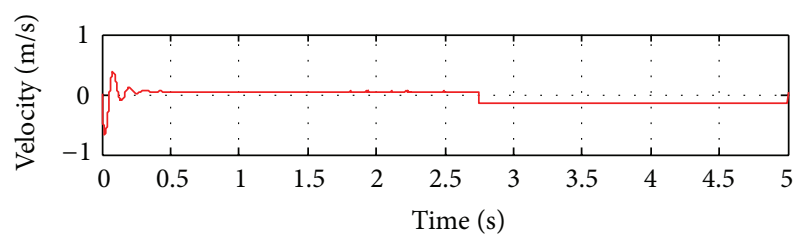

(b)

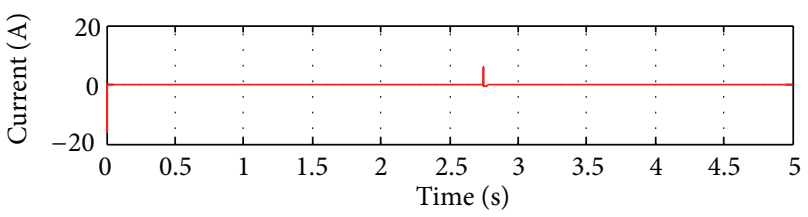

(c)

FIGURE 4: Residual outputs when accelerometer failed.

not sensitive to the disturbance caused by the induced delay of random network within certain range.

Assume that the acceleration sensor is unexpectedly damaged at $8 \mathrm{~s}$, by which a constant fault signal with the amplitude of 0.2 is superimposed on the normal signal. Figure 4 shows the output of the robust fault observer.

From Figure 4, it can be concluded that when the accelerometer is abruptly faulted at $2.75 \mathrm{~s}$, the speed direction

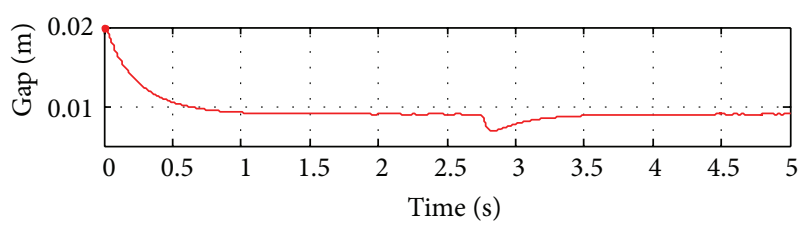

(a)

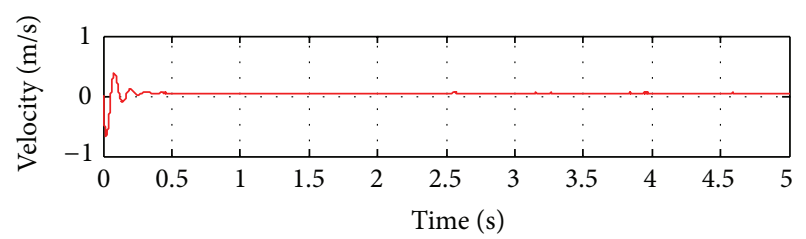

(b)

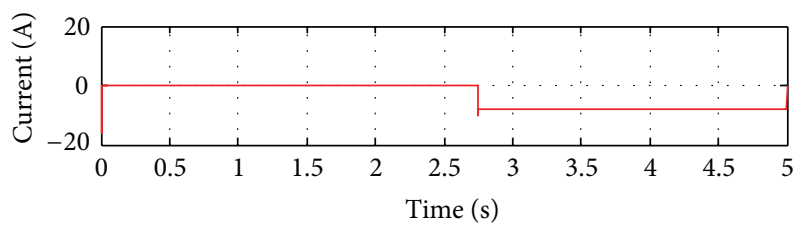

(c)

FIGURE 5: Residual outputs when current sensors failed.

TABLE 1: Parameters of suspension control system.

\begin{tabular}{lc}
\hline Property & Value \\
\hline$k$ & 0.000693 \\
$m$ & $750 \mathrm{~kg}$ \\
$g$ & $9.81 \mathrm{~m} / \mathrm{s}^{2}$ \\
$z_{\text {eq }}$ & $0.02 \mathrm{~m}$ \\
$R$ & $0.5 \Omega$ \\
$L$ & $0.6 \mathrm{H}$ \\
\hline
\end{tabular}

of residual output responds to the fault signal immediately. Meanwhile, the fault can be judged according to the phase step. When the accelerometer failed, the system has no impact on the gap and current channel. It shows a good directivity and can be judged that accelerometer faults have occurred.

Similarly, the system can detect the current faults in an excellent way. As shown in Figure 5, when a constant fault signal of $8 \mathrm{~A}$ is superimposed on the current sensors, it can be analyzed that the faults come from the current sensors from the residual signal. Thereby, the fault detection can be completed. The simulation results indicate that the fault detection approach is effective.

\section{Conclusion}

In engineering practice, high security and reliability of the suspension control system are the most foundational requirements for the safety operation of Maglev train. In this paper, the sensors fault detection of the networked suspension control system with random bounded time delay is studied. In order to realize the real states' estimation of the networked suspension control system with arbitrary 
precision under the conditions of random bounded time delay, a nonlinear adaptive robust observer is presented and well designed. And the residual between the real states and the observer outputs indicates which kind of sensor failures occurs. Finally, simulations results demonstrate the effectiveness of this proposed method.

\section{Acknowledgment}

This work is supported by the National Natural Science Foundation of China under Grant no. 11202230.

\section{References}

[1] L. G. Yan, "Progress of the maglev transportation in china," IEEE Transactions on Applied Superconductivity, vol. 16, no. 2, pp. 1138-1141, 2006.

[2] C. Wensen, "Maglev technology development and automatic control," in Proceedings of the 22th Chinese Control Conference, vol. 8, pp. 27-30, Yichang, China, 2003.

[3] L. Yun and L. Zhiqiang, "Design and realization of suspension system based on networked control systems for maglev train," Journal of System Simulation, vol. 21, no. 14, pp. 4420-4425, 2009.

[4] H. Guang, L. Yun, L. Z. Long, and J. I. Zhide, "Research on fault tolerant control technology based on networked control system of Maglev train," in Proceddings of the International Conference on Intelligent System Design and Engineering Application (ISDEA '10), 2010.

[5] L. Zhiqiang, H. Aming, and C. Chengkan, "Sensitivity constrained robust controller design of suspension controller for Maglev train," Journal of Control Theory and Applications, vol. 21, no. 3, pp. 804-808, 2004.

[6] Z. Zhizhou, L. Xiaolong, and L. Zhiqiang, "Sensor fault tolerance method for Maglev train based on state-observer," Electric Drive for Locomotives, no. 4, pp. 39-42, 2008.

[7] J. P. Hespanha, P. Naghshtabrizia, and X. Yonggang, "Survey of recent results in networked control systems," Proceedings of the IEEE, vol. 95, no. 1, pp. 138-162, 2007.

[8] L. Zhang, Y. Shi, T. Chen et al., "A new method for stabilization of networked control systems with random delays," IEEE Transactions on Automatic Control, vol. 50, no. 8, pp. 1177-1181, 2005.

[9] X. M. Zhang, G. P. Lu, and Y. E. Zheng, "Stabilization of networked stochastic time delay fuzzy systems with data dropout," IEEE Transactions on Fuzzy Systems, vol. 16, no. 3, pp. 798-807, 2008.

[10] H. G. Zhang, J. Yang, C. Y. Su et al., "T-S fuzzy modelbased robust $H_{\infty}$ design for networked control systems with uncertainties," IEEE Transactions on Industrial Informatics, vol. 3, no. 4, pp. 289-301, 2007.

[11] Y. Q. Wang, H. Ye, X. S. Ding, and G. Z. Wang, "Fault detection of networked control systems based on optimal robust fault detection filter," Acta Automatica Sinica, vol. 34, no. 12, pp. 15341539, 2008.

[12] B. Liu, Y. Xia, Y. Yang, and M. Fu, "Robust fault detection of linear systems over networks with bounded packet loss," Journal of the Franklin Institute, vol. 349, no. 7, pp. 2480-2499, 2012.

[13] J. Zhang, L. Du, M. Ren, and G. Hou, "Minimum error entropy filter for fault detection of networked control systems," Entropy, no. 14, pp. 505-516, 2012.
[14] C. Peng, M. R. Fei, and E. Tian, "Networked control for a class of T-S fuzzy systems with stochastic sensor faults," Fuzzy Sets and Systems, vol. 212, pp. 62-77, 2013.

[15] B. Jiang, P. Shi, and Z. Mao, "Sliding mode observer-based fault estimation for nonlinear networked control systems," Circuits, Systems, and Signal Processing, vol. 30, no. 1, pp. 1-16, 2011.

[16] H. He, X. Dexia, H. Xiaodong, Z. Dengfeng, and W. Zhiquan, "Fault detection for a class of T-S Fuzzy model based nonlinear networked control system," Information and Control, vol. 38, no. 6, pp. 703-710, 2009.

[17] M. Y. Zhong, Y. X. Liu, and C. E. Ma, "Observer-based fault detection for networked control systems with random time delays," in Proceedings of the 1st International Conference on Innovative Computing, Information and Control, pp. 528-531, Los Alamitos, CA, USA, 2006.

[18] M. Y. Zhong and Q. L. Hart, "Fault detection filter design for a class of networked control systems," in Proceedings of the 6th World Congress on Intelligent Control and Automation, pp. 215219, Piscataway, NJ, USA, 2006.

[19] Q. Zong, F. Zeng, W. Liu, Y. Ji, and Y. Tao, "Sliding mode observer-based fault detection of distributed networked control systems with time delay," Circuits, Systems, and Signal Processing, vol. 31, no. 1, pp. 203-222, 2012.

[20] E. Tian, C. Peng, and Z. Gu, "Fault tolerant control for discrete networked control systems with random faults," International Journal of Control, Automation, and Systems, vol. 10, no. 2, pp. 444-448, 2012.

[21] E. Tian, D. Yue, T. C. Yang, Z. Gu, and G. Lu, “T-S fuzzy modelbased robust stabilization for networked control systems with probabilistic sensor and actuator failure," IEEE Transactions on Fuzzy Systems, vol. 19, no. 3, pp. 553-561, 2011.

[22] E. Tian and D. Yue, "Reliable $H_{\infty}$ filter design for T-S fuzzy model-based networked control systems with random sensor failure," International Journal of Robust and Nonlinear Control, vol. 23, no. 1, pp. 15-32, 2013.

[23] Y. Liu, "Robust adaptive observer for nonlinear systems with unmodeled dynamics," Automatica, vol. 45, no. 8, pp. 1891-1895, 2009.

[24] Y. Jose, M. Pau, and M. F. Josep, "Control loop performance analysis over networked control systems," in IEEE 28th Annual Conference of the Industrial Electronics Society, pp. 2880-2885, 2002.

[25] W. Jun, L. Xiaolong, and L. Zhiqiang, "The analysis and measure of the real-time performance on CAN bus control network," Industry Control Computer, vol. 17, no. 10, pp. 21-23, 2004.

[26] A. Yetendje, M. M. Seron, J. A. De Doná, and J. J. Martínez, "Sensor fault-tolerant control of a magnetic levitation system," International Journal of Robust and Nonlinear Control, vol. 20, no. 18, pp. 2108-2121, 2010.

[27] L. Xi-mai, W. Wan-yun, W. Ke-tai, and Y. Fei, "Fault diagnosis of network control systems with uncertain time-delay," Systems Engineering and Electronics, vol. 30, no. 4, pp. 767-771, 2008.

[28] J. Xiuqin, Observer Design and PerFormance Analysis For Nonlinear Systems, Shandong University, Jinan, China, 2008. 


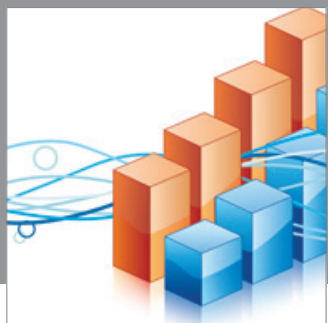

Advances in

Operations Research

mansans

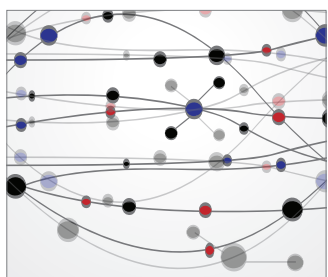

The Scientific World Journal
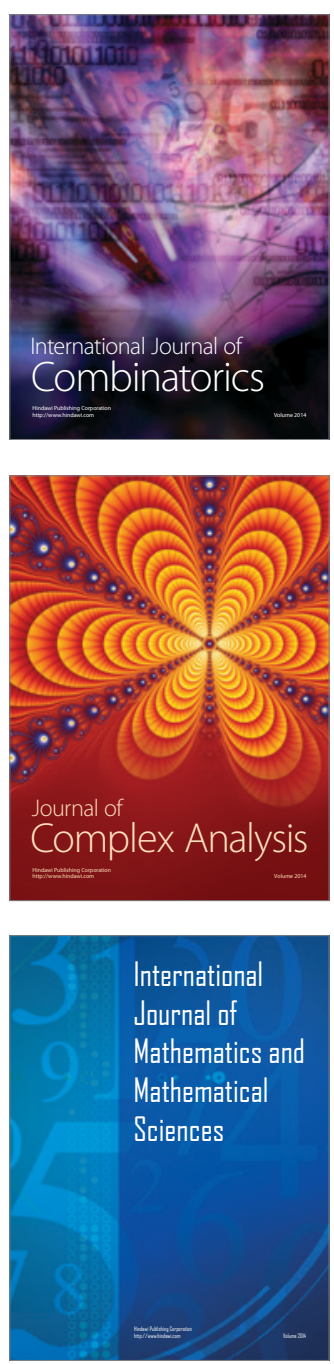
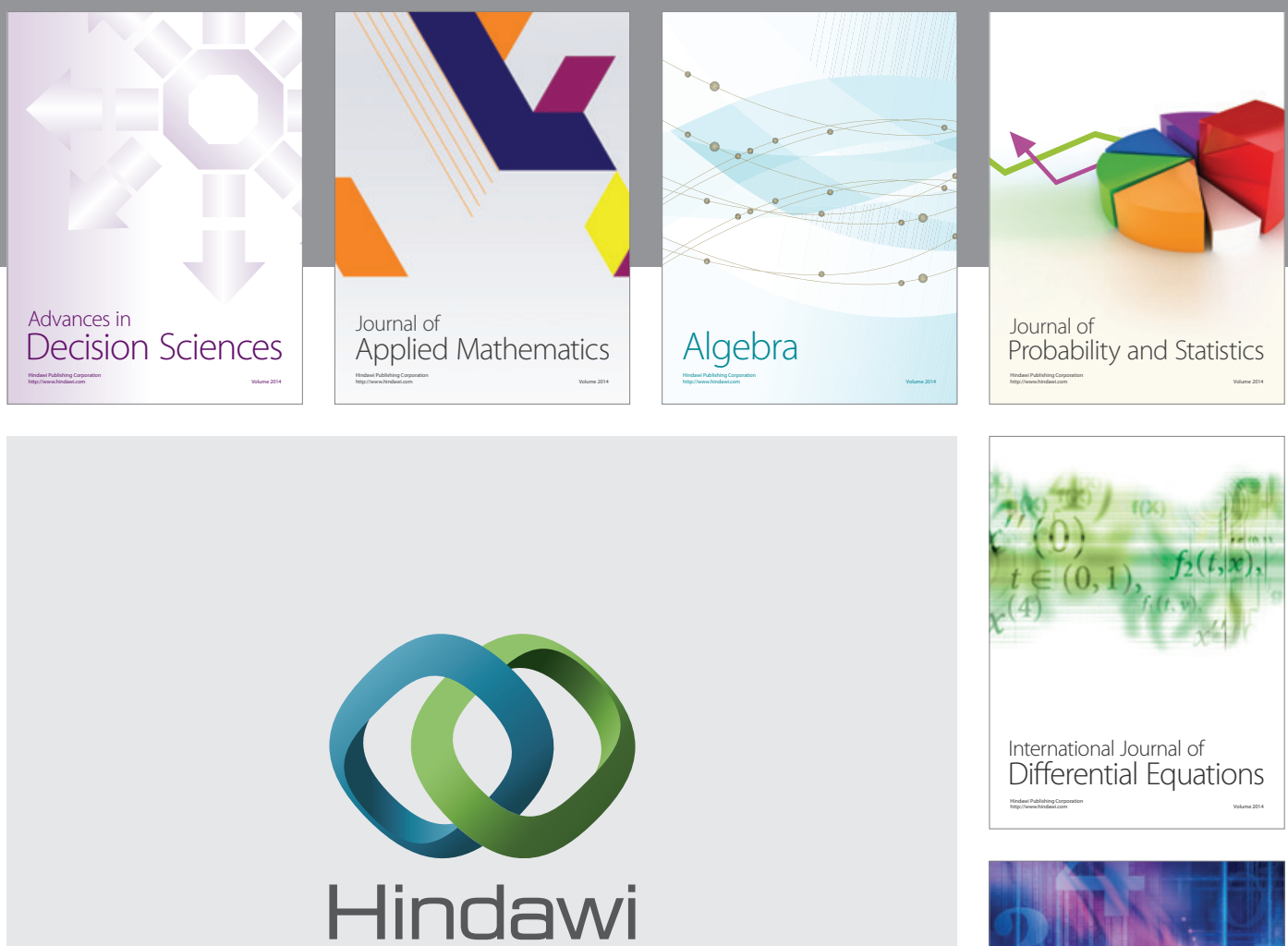

Submit your manuscripts at http://www.hindawi.com
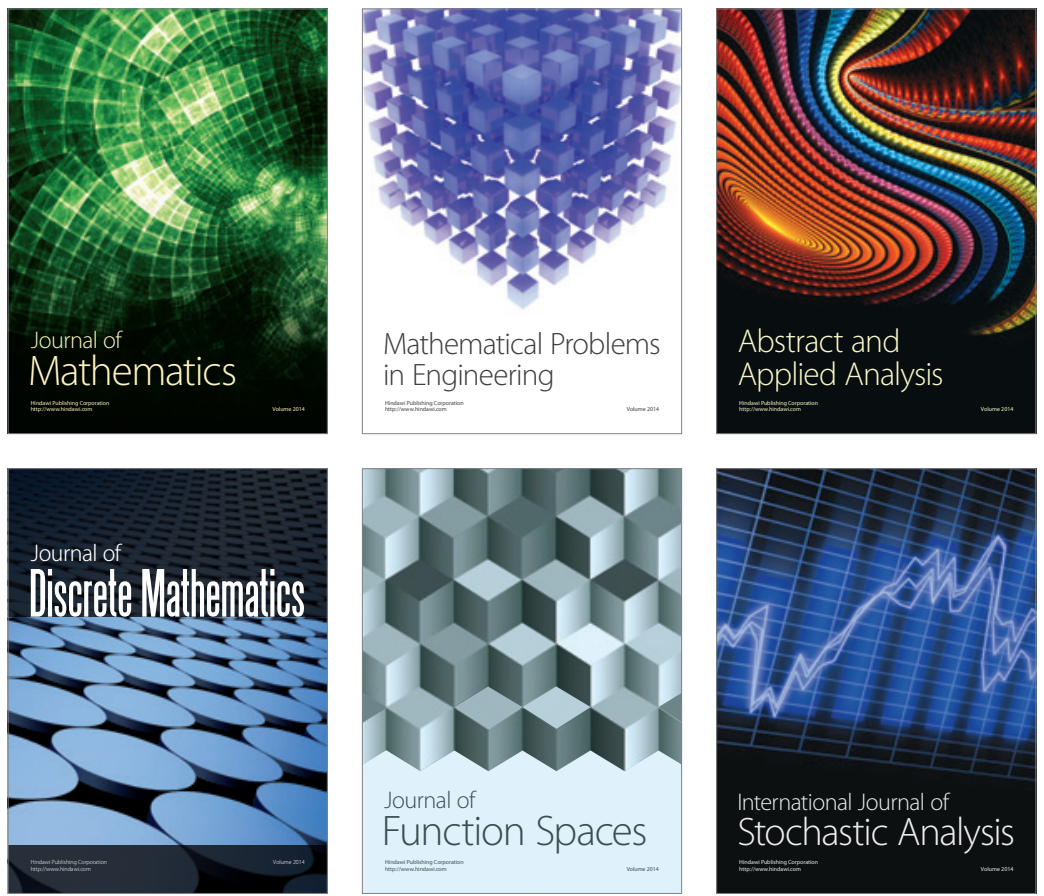

Journal of

Function Spaces

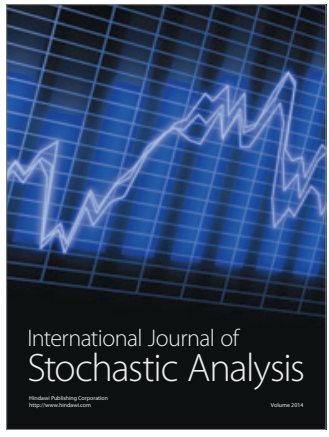

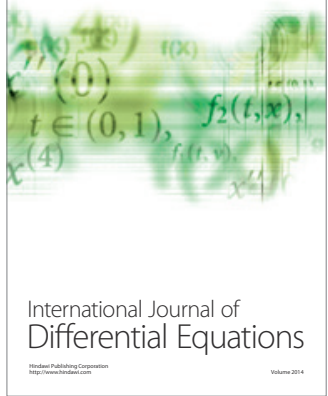
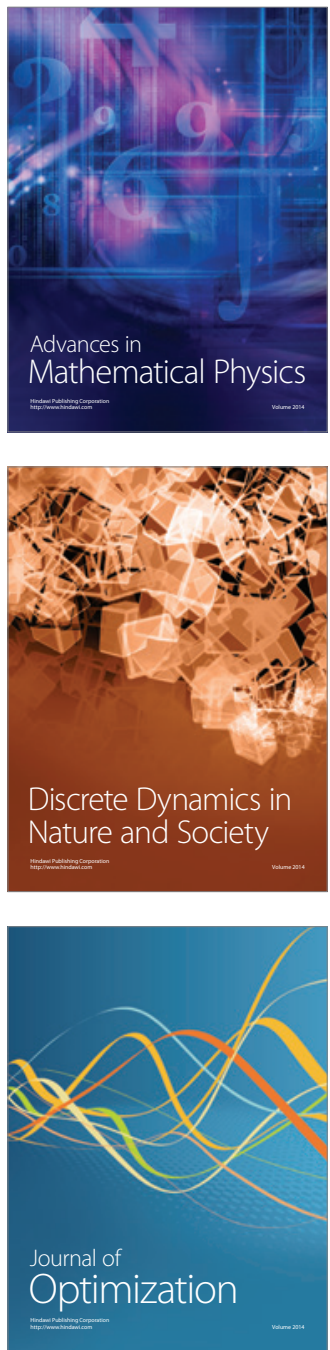